(C)2009 IEEE. Personal use of this material is permitted. However, permission to reprint/republish this material for advertising or promotional purposes or for creating new collective works for resale or redistribution to servers or lists, or to reuse any copyrighted component of this work in other works must be obtained from the IEEE. 


\title{
Input-to-State Stability for A Class of Hybrid Dynamical Systems via Hybrid Time Approach
}

\author{
Bin Liu, David J. Hill, Kok Lay Teo
}

\begin{abstract}
This paper studies the ISS (input-to-state stability) for a class of HDS (hybrid dynamical systems). By using the concept of hybrid time for HDS, two kinds of ISS notions are proposed. They are called the first ISS property and the second ISS property of HDS. By employing the ISS properties on continuous and/or discrete dynamics in the HDS, the first and second ISS properties for the whole HDS are investigated. We show that there exists the first ISS property for the whole HDS if any one of dynamics in the HDS has the ISS property and the dwell time on this dynamics is sufficiently long even if there is no ISS property for the other dynamics. Moreover, the second ISS property of HDS is derived for the cases in which one kind of dynamics without external inputs has a stability property while the other one has no stability and ISS properties. Finally, one example is given for illustration.
\end{abstract}

\section{INTRODUCTION}

ISS (input-to-state stability) analysis of nonlinear systems aims to investigate how external disturbances affect the system stability. Since the notion of ISS was proposed in [1] in the late 1980s, ISS property analysis of nonlinear systems with external disturbances has quickly become one of the more active research topics in nonlinear analysis and design. ISS has been successfully employed in the stability analysis and control synthesis of nonlinear systems. These works mainly focused on the following topics related to the study of the ISS property: feedback ISS stabilization for nonlinear systems [2, 4, 9-10, 13], ISS nonlinear small gain results for nonlinear systems $[6,17,18]$, ISS for sampleddata systems [8], ISS problem using averaging technique [7], ISS properties of networked control systems [5], ISS for discrete-time systems [3], ISS for impulsive systems in [11-12,37], and ISS for time-delay systems in [14-16,39].

Hybrid dynamical systems (HDS) provide a natural framework for mathematical modeling of many physical phenomena and practical engineering problems. Their study has received considerable attention for the last two decades, see $[26-36,38]$ and the references cited therein. Due to many factors such as the hybrid structure, multiple dynamics, timedelays, and external disturbances, etc, it is now recognized that the dynamical behaviors (for example, stability and ISS)

This work was supported by the Australian Research Council Discovery Project Scheme (No. DP0881391) and NSFC-China (No. 60874025).

B. Liu is with the Department of Information Engineering, The Australian National University, ACT 0200, Australia. Bin. Liu@anu. edu. au

D. J. Hill is with the Department of Information Engineering, The Australian National University, ACT 0200, Canberra, Australia. David.Hilleanu.edu.au

K. L. Teo is with the Department of Mathematics and Statistics, Curtin University of Technology, Perth, West Australia, Australia. K.L.Teo@curtin.edu.au for HDS may be complex and hence it remains a difficult task to investigate their dynamics despite progress so far.

Recently, stability and $\mathscr{K} \mathscr{L} \mathscr{L}$-stability properties based on set-value stability theory, invariance principles, and smooth Lyapunov functions are investigated for more general HDS in the literature, see [20-25]. In these references, concepts such as hybrid time domain, hybrid trajectory for HDS were proposed. It has been shown that these concepts of HDS contribute to the analysis of stability for HDS. The mentioned research on the stability of HDS assumes the absence of external disturbance inputs. More recently, ISS analysis for HDS has been reported in [19] by using $\mathscr{K} \mathscr{L} \mathscr{L}$ functions and smooth ISS-Lyapunov function method. However, the ISS property studied in [19] requires that every subsystem in a HDS has the corresponding ISS property and thus it can not reflect completely the diversity of dynamics for subsystems in a HDS. Hence, the ISS of HDS has not been fully developed.

In this paper, we focus on the study of ISS for a class of HDS. In the HDS, there exist two kinds of dynamics (continuous-time and discrete-time dynamics). And these two kinds of dynamics appear alternately. The continuous-time dynamics can be looked upon as the natural process of HDS while the discrete-time dynamics is due to data-sampling or discrete control inputs. In [37], we have noted that the hybrid time variables, specially the discrete-time variable in the hybrid time domain, play an important role in the analysis of ISS for HDS. On account of this fact, in this paper, we propose two kinds of ISS notions for HDS, i.e., the first and the second ISS property of HDS. These two ISS notions are more general than that in [19]. By means of ISS properties allocating to the different types of dynamics in the HDS, sufficient conditions for the first and second ISS property of such HDS are established.

\section{PReliminaries}

In the sequel, let $R=(-\infty, \infty), R_{+}=[0,+\infty), R^{n}$ be the $n$-dimensional Euclidean space, and $N=\{0,1,2, \cdots\}$. For $t_{1}, t_{2} \in R_{+}$, satisfying $t_{1} \leq t_{2}$ and $t_{2}-t_{1} \in N$, denote $\mathscr{N}\left[t_{1}, t_{2}\right]=\left\{t_{1}+k: k \in N, t_{1} \leq t_{1}+k \leq t_{2}\right\}$.

A function $\gamma: R_{+} \rightarrow R_{+}$is of class- $\mathscr{K}(\gamma \in \mathscr{K})$ if it is continuous, zero at zero and strictly increasing. It is of class$\mathscr{K}_{\infty}$ if it is of class- $\mathscr{K}$ and is unbounded. A continuous function $\beta: R_{+} \times R_{+} \rightarrow R_{+}$is of class- $\mathscr{K} \mathscr{L}$ if $\beta(\cdot, t)$ is of class- $\mathscr{K}$ for $t \geq 0$ and $\beta(s, \cdot)$ is monotonically decreasing to zero for $s>0$. A continuous function $\hat{\beta}: R_{+} \times R_{+} \rightarrow R_{+}$is of class- $\mathscr{K} \mathscr{K}$ if $\hat{\beta}(\cdot, t)$ is of class- $\mathscr{K}$ for $t \geq 0$ and $\hat{\beta}(s, \cdot)$ is of class- $\mathscr{K}$ for $s \geq 0$. A function $\beta: R_{+} \times N \rightarrow R_{+}$is 
of class- $\mathscr{K} \mathscr{D}$ if $\beta(\cdot, i) \in \mathscr{K}$ for $i \in N$, and for any given $s>0$, the series $\{\beta(s, i), i \in N\}$ is monotonically decreasing to zero.

Denote: $\mathscr{K}^{+} \triangleq\{\gamma \in \mathscr{K}: \gamma(a+b) \leq \gamma(a)+\gamma(b), a, b \in$ $\left.R_{+}\right\}, \quad \mathscr{K}^{+} \mathscr{L} \triangleq\{\beta \in \mathscr{K} \mathscr{L}: \beta(a+b, t) \leq \beta(a, t)+$ $\left.\beta(b, t), a, b \in R_{+}\right\}, \mathscr{K}^{+} \mathscr{L}^{+} \triangleq\left\{\beta \in \mathscr{K}^{+} \mathscr{L}: \beta(\beta(a, s), t) \leq\right.$ $\left.\beta(a, t+s), a, s, t \in R_{+}\right\}$, and $\mathscr{K}^{+} \mathscr{K} \triangleq\{\alpha \in \mathscr{K} \mathscr{K}: \alpha(a+$ $\left.b, t) \leq \alpha(a, t)+\alpha(b, t), a, b \in R_{+}\right\}$.

Given a $n \times n$ matrix $A,\|A\|$ denotes the norm of $A$ induced by the Euclidean vector norm, i.e., $\|A\|=\left[\lambda_{\max }\left(A^{T} A\right)\right]^{\frac{1}{2}}$. For any function $u: R_{+} \rightarrow R^{n}$, we denote $\|u\|_{[t]}=\sup \left\{\|u(s)\|: 0 \leq s \leq t, s \in R_{+}\right\}$, and $\|u\|_{\infty}=\sup \left\{\|u(s)\|: s \in R_{+}\right\}$.

Consider the HDS under external inputs:

$$
\left\{\begin{array}{l}
x(t) \in S_{c i}=\left\{x(t): \dot{x}=f_{c i}\left(t, x, w_{c i}\right)\right\}, t \in I_{c i}, \\
x(t) \in S_{d i}=\left\{x(t): x(t)=f_{d i}\left(t-1, x(t-1), w_{d i}\right)\right\}, t \in I_{d i}, \\
x\left(t_{0}\right)=x_{0}, \quad i \in N,
\end{array}\right.
$$

where $x(t) \in R^{n}$ is the state of HDS; $w_{c i}: R_{+} \rightarrow R^{n_{c}}, w_{d i}$ : $R_{+} \rightarrow R^{n_{d i}}$, with $w_{c i}(0)=0, w_{d i}(0)=0$, represent the external disturbance inputs satisfying $\left\|w_{c}\right\|_{\infty}<\infty,\left\|w_{d}\right\|_{\infty}<\infty$, where $\left\|w_{c}\right\|_{\infty} \triangleq \sup _{i \in N}\left\{\left\|w_{c i}\right\|_{\infty}\right\},\left\|w_{d}\right\|_{\infty} \triangleq \sup _{i \in N}\left\{\left\|w_{d i}\right\|_{\infty}\right\}$; $I_{c i}=\left[t_{2 i}, t_{2 i+1}\right], I_{d i}=\mathscr{N}\left[t_{2 i+1}+1, t_{2(i+1)}\right], i \in N$, stand for the continuous and discrete time intervals with $\lim _{i \rightarrow \infty} t_{i}=+\infty$.

For $i \in N$, denote $\mathscr{I}_{i}=\left(I_{c i} \cup I_{d i},\{i\}\right)$. We call $\mathscr{I}_{i}$ a hybrid time domain. For any $(t, j) \in \mathscr{I}_{i}$, i.e., $t \in I_{c i} \cup I_{d i}, j=i$, we call the $t$ as the first time variable and the $j$ as the second time variable.

Under the external input $\left(w_{c i}, w_{d i}\right), i \in N$, we denote $x(t, i) \triangleq x\left((t, i), t_{0}, x_{0}, w_{c}, w_{d}\right),(t, i) \in \mathscr{I}_{i}, i \in N$, as the solution to system (1). Since $x\left(t_{2 i}, i\right)$ is the start point on $\mathscr{I}_{i}$ and $x\left(t_{2 i}, i-1\right)$ is the end point on $\mathscr{I}_{i-1}$, thus, $x\left(t_{2 i}, i\right)=$ $x\left(t_{2 i}, i-1\right)$ for any $i \geq 1, i \in N$. For any $(t, i) \in \mathscr{I}_{i}, i \in N$, denote $\left\|w_{c}\right\|_{[t]}=\sup _{0 \leq j \leq i}\left\{\left\|w_{c j}(s)\right\|: 0 \leq s \leq t\right\},\left\|w_{d}\right\|_{[t]}=$ $\sup _{0 \leq j \leq i}\left\{\left\|w_{d j}(s)\right\|: 0 \leq s \leq t\right\}$.

Definition 2.1. (i) The HDS (1) is said to have the first ISS property if there exist a function $\beta \in \mathscr{K} \mathscr{L}$, and functions $\varphi, \gamma_{c}, \gamma_{d} \in \mathscr{K}$ such that, for any $(t, i) \in \mathscr{I}_{i}, i \in N, t \geq t_{0}$,

$$
\varphi(\|x(t, i)\|) \leq \beta\left(\left\|x_{0}\right\|, t-t_{0}\right)+\gamma_{c}\left(\left\|w_{c}\right\|_{[t]}\right)+\gamma_{d}\left(\left\|w_{d}\right\|_{[t]}\right) .
$$

(ii) The HDS (1) is said to have the second ISS property if there exist a function $\beta \in \mathscr{K} \mathscr{D}$, and functions $\varphi, \gamma_{c}, \gamma_{d} \in \mathscr{K}$ such that, for any $(t, i) \in \mathscr{I}_{i}, i \in N$,

$$
\varphi(\|x(t, i)\|) \leq \beta\left(\left\|x_{0}\right\|, i\right)+\gamma_{c}\left(\left\|w_{c}\right\|_{[t]}\right)+\gamma_{d}\left(\left\|w_{d}\right\|_{[t]}\right) .
$$

(iii) The HDS (1) is said to have the first exponential ISS property if there exist constants $\alpha>0, K>0$, and $\mathscr{K}$ functions $\gamma_{c}$ and $\gamma_{d}$, such that for any $(t, i) \in \mathscr{I}_{i}, i \in N, t \geq t_{0}$,

$$
\|x(t, i)\| \leq K e^{-\alpha\left(t-t_{0}\right)}\left\|x_{0}\right\|+\gamma_{c}\left(\left\|w_{c}\right\|_{[t]}\right)+\gamma_{d}\left(\left\|w_{d}\right\|_{[t]}\right) .
$$

(iv) The HDS (1) is said to have the second exponential ISS property if there exist constants $\alpha>0, K>0$, and $\mathscr{K}$ functions $\gamma_{c}$ and $\gamma_{d}$, such that for any $(t, i) \in \mathscr{I}_{i}, i \in N$,

$$
\|x(t, i)\| \leq K e^{-\alpha i}\left\|x_{0}\right\|+\gamma_{c}\left(\left\|w_{c}\right\|_{[t]}\right)+\gamma_{d}\left(\left\|w_{d}\right\|_{[t]}\right) .
$$

Remark 2.1. (i) Noting that for any $(t, i) \in \mathscr{I}_{i}$, we have $t \rightarrow \infty$ if and only if $i \rightarrow \infty$. Hence, $\lim _{t \rightarrow \infty} \beta\left(\left\|x_{0}\right\|, t-t_{0}\right)=$ $\lim _{i \rightarrow \infty} \beta\left(\left\|x_{0}\right\|, i\right)=0$ and hence Definition 2.1 (ii) and (iv) are well-defined.

(ii) In very recent literature [19], the authors investigated the ISS issue for hybrid systems by using the class- $\mathscr{K} \mathscr{L} \mathscr{L}$ function. In [19], the ISS is defined for an nonempty compact $\mathscr{A}$ of state space as

$$
\omega(x(t, j)) \leq \max \left\{\gamma\left(\omega\left(x_{0}\right), t, j\right), \kappa\left(\|u\|_{(t, j)}\right\}, \forall(t, j) \in \operatorname{dom} x,\right.
$$

where $\gamma \in \mathscr{K} \mathscr{L} \mathscr{L}, \kappa \in \mathscr{K}$, and $\omega$ is a proper indicator of set $\mathscr{A}$. It should be noted that, since $\gamma \in \mathscr{K} \mathscr{L} \mathscr{L}$, we have $\lim _{t \rightarrow \infty} \gamma\left(\omega\left(x_{0}\right), t, j\right)=\lim _{j \rightarrow \infty} \gamma\left(\omega\left(x_{0}\right), t, j\right)=0$. Hence, compared with the above ISS notions in Definition 2.1, ISS studied in [19] is one kind of more strong ISS property for hybrid systems.

Lemma 2.1. Let $\alpha \in \mathscr{K}$. If the Dini derivative

$$
D^{+} m(t) \leq \alpha(m(t)), \text { for all } t \geq t_{0}, t \in R_{+},
$$

then there exists $\beta \in \mathscr{K} \mathscr{K}$ with $\beta(s, 0) \geq s$ such that

$$
m(t) \leq \beta\left(m_{0}, t-t_{0}\right), \quad t \geq t_{0}, t \in R_{+} .
$$

where $m_{0}=m\left(t_{0}\right)$.

Proof. The proof is similar to that in Lemma 6.1 of [1]. The detailed proof is omitted due to the limited space.

\section{Two KINDS OF ISS PROPERTIES OF HDS}

In this section, the first and second ISS properties are investigated for HDS (1).

\section{A. The First ISS Property of HDS}

In this part, three kinds of the first ISS properties are investigated according to the different ISS properties of $S_{c i}$ and $S_{d i}$ in the whole HDS (1).

Denote $\Delta_{c i}=t_{2 i+1}-t_{2 i}, \Delta_{d i}=t_{2(i+1)}-t_{2 i+1}, \quad \Delta_{i}^{-}=$ $\Delta_{c i}+\Delta_{d i-1}, \quad \Delta_{i}^{+}=\Delta_{c i}+\Delta_{d i}$, for any $i \in N ; \quad \Delta_{i n f} \triangleq$ $\inf _{i \in N}\left\{\Delta_{c i}, \Delta_{d i}\right\}, \Delta_{\text {sup }} \triangleq \sup _{i \in N}\left\{\Delta_{c i}, \Delta_{d i}\right\} ; \Delta_{c}^{i n f}=\inf _{i \in N}\left\{\Delta_{c i}\right\}$, $\Delta_{d}^{\text {inf }}=\inf _{i \in N}\left\{\Delta_{d i}\right\} ; \Delta_{c}^{\text {sup }}=\sup _{i \in N}\left\{\Delta_{c i}\right\}, \Delta_{d}^{\text {sup }}=\sup _{i \in N}\left\{\Delta_{d i}\right\}$.

Case I: All $S_{c i}$ and $S_{d i}$ have ISS properties.

Theorem 3.1. Assume the following conditions hold:

(i) there exist $\beta_{c}, \beta_{d} \in \mathscr{K}^{+} \mathscr{L}^{+}$and $\gamma_{c}, \gamma_{d} \in \mathscr{K}$ such that

$$
\begin{aligned}
\|x(t, i)\| & \leq \beta_{c}\left(\left\|x\left(t_{2 i}, i\right)\right\|, t-t_{2 i}\right)+\gamma_{c}\left(\left\|w_{c}\right\|_{[t]}\right), t \in I_{c i} ; \\
\|x(t, i)\| & \leq \beta_{d}\left(\left\|x\left(t_{2 i+1}, i\right)\right\|, t-t_{2 i+1}\right) \\
& +\gamma_{d}\left(\left\|w_{d}\right\|_{[t]}\right), t \in I_{d i} ;
\end{aligned}
$$

where functions $\beta_{c}, \beta_{d}$ satisfy, for any $a, s, t \in R_{+}$,

$$
\begin{aligned}
& \max \left\{\beta_{c}\left(\beta_{d}(a, s), t\right), \beta_{d}\left(\beta_{c}(a, s), t\right)\right\} \\
& \quad \leq \max \left\{\beta_{c}(a, s+t), \beta_{d}(a, s+t)\right\} ;
\end{aligned}
$$

(ii) for any $a \in R_{+}$, there exists $\mathscr{K}$-class function $\hat{\gamma}$ such that

$$
\sum_{j=0}^{i} \beta\left(a, t_{2 i}-t_{2 j}\right) \leq \hat{\gamma}(a), \quad i \in N,
$$

where $\beta(a, s)=\max \left\{\beta_{c}(a, s), \beta_{d}(a, s)\right\}$ for any $a, s \in R_{+}$. Then, the HDS (1) has the first ISS property. 
Proof. First, by the following Claims $1^{\circ}-2^{\circ}$, we claim that $\beta \in \mathscr{K}^{+} \mathscr{L}^{+}$.

Claim 1 $1^{\circ}$ : if $\beta_{c}, \beta_{d} \in \mathscr{K}^{+} \mathscr{L}$, then, $\beta \in \mathscr{K}^{+} \mathscr{L}$.

Claim $2^{\circ}$ : if $\beta_{c}, \beta_{d} \in \mathscr{K}^{+} \mathscr{L}^{+}$, then, $\beta \in \mathscr{K}^{+} \mathscr{L}^{+}$.

Thus, from conditions (i)-(ii) and $\beta_{c}, \beta_{d}, \beta \in \mathscr{K}^{+} \mathscr{L}^{+}$and noting that $x\left(t_{2 i}, i\right)=x\left(t_{2 i}, i-1\right)$, it yields, for any $i \in N$,

$$
\begin{aligned}
& \left\|x\left(t_{2 i+1}, i\right)\right\| \leq \beta_{c}\left(\left\|x\left(t_{2 i}, i\right)\right\|, \Delta_{c i}\right)+\gamma_{c}\left(\left\|w_{c}\right\|_{\left[t_{2 i+1}\right]}\right) \\
& \leq \beta_{c}\left(\beta_{d}\left(\left\|x\left(t_{2 i-1}, i-1\right)\right\|, \Delta_{d i-1}\right)+\gamma_{d}\left(\left\|w_{d}\right\|_{\left[t_{2 i}\right]}\right), \Delta_{c i}\right) \\
& \quad+\gamma_{c}\left(\left\|w_{c}\right\|_{\left[t_{2 i+1}\right]}\right) \\
& \leq \beta_{c}\left(\beta_{d}\left(\left\|x\left(t_{2 i-1}, i-1\right)\right\|, \Delta_{d i-1}\right), \Delta_{c i}\right) \\
& \quad+\beta_{c}\left(\gamma_{d}\left(\left\|w_{d}\right\|_{\left[t_{2 i}\right]}\right), \Delta_{c i}\right)+\gamma_{c}\left(\left\|w_{c}\right\|_{\left[t_{2 i+1}\right]}\right) \\
& \leq \beta\left(\left\|x\left(t_{2 i-1}, i-1\right)\right\|, \Delta_{i}^{-}\right)+\beta_{c}\left(\gamma_{d}\left(\left\|w_{d}\right\|_{\left[t_{2 i}\right]}\right), \Delta_{c i}\right) \\
& \quad+\gamma_{c}\left(\left\|w_{c}\right\|_{\left[t_{2 i+1}\right]}\right) .
\end{aligned}
$$

Let $a_{i}=\left\|x\left(t_{2 i+1}, i\right)\right\| \quad$ and $\quad b_{i}=\beta\left(\gamma_{d}\left(\left\|w_{d}\right\|_{\left[t_{2 i}\right]}\right), \Delta_{c i}\right)+$ $\gamma_{c}\left(\left\|w_{c}\right\|_{\left[t_{2 i+1}\right]}\right), i \in N$, then,

$$
a_{i} \leq \beta\left(a_{i-1}, \Delta_{i}^{-}\right)+b_{i}, i \in N .
$$

By induction and the property of function $\beta$, we get

$$
\begin{aligned}
& a_{i} \leq \beta\left(a_{0}, t_{2 i+1}-t_{1}\right)+\sum_{j=1}^{i} \beta\left(\gamma_{d}\left(\left\|w_{d}\right\|_{\left[t_{2 i}\right]}\right), t_{2 i+1}-t_{2 j}\right) \\
& +\sum_{j=1}^{i-1} \beta\left(\gamma_{c}\left(\left\|w_{c}\right\|_{\left[t_{2 i+1}\right]}\right), t_{2 i+1}-t_{2 j+1}\right)+\gamma_{c}\left(\left\|w_{c}\right\|_{\left[t_{2 i+1}\right]}\right) .
\end{aligned}
$$

Noting $a_{0}=\left\|x\left(t_{1}, 0\right)\right\| \leq \beta_{c}\left(\left\|x_{0}\right\|, t_{1}-t_{0}\right)+\gamma_{c}\left(\left\|w_{c}\right\|_{\left[t_{1}\right]}\right)$, and thus by (12), it yields that

$$
\begin{aligned}
a_{i} & \leq \beta\left(\left\|x_{0}\right\|, t_{2 i+1}-t_{0}\right)+\sum_{j=1}^{i} \beta\left(\gamma_{d}\left(\left\|w_{d}\right\|_{\left[t_{2 i}\right]}\right), t_{2 i+1}-t_{2 j}\right) \\
& +\sum_{j=1}^{i-1} \beta\left(\gamma_{c}\left(\left\|w_{c}\right\|_{\left[t_{2 i+1}\right]}\right), t_{2 i+1}-t_{2 j+1}\right) \\
& +\beta\left(\gamma_{c}\left(\left\|w_{c}\right\|_{\left[t_{2 i+1}\right]}\right), t_{2 i+1}-t_{1}\right)+\gamma_{c}\left(\left\|w_{c}\right\|_{\left[t_{2 i+1}\right]}\right) .
\end{aligned}
$$

Hence, for any $(t, i) \in \mathscr{I}_{i}$, if $t \in I_{c i}=\left[t_{2 i}, t_{2 i+1}\right]$, then, by condition (i) and (13), we get

$$
\begin{aligned}
& \|x(t, i)\| \leq \beta\left(\left\|x_{0}\right\|, t-t_{0}\right)+\sum_{j=1}^{i} \beta\left(\gamma_{d}\left(\left\|w_{d}\right\|_{\left[t_{2 i}\right]}\right), t-t_{2 j}\right) \\
& +\sum_{j=0}^{i-1} \beta\left(\gamma_{c}\left(\left\|w_{c}\right\|_{\left[t_{2 i+1}\right]}\right), t-t_{2 j+1}\right)+\gamma_{c}\left(\left\|w_{c}\right\|_{[t]}\right) \\
& \leq \beta\left(\left\|x_{0}\right\|, t-t_{0}\right)+\sum_{j=1}^{i} \beta\left(\gamma_{d}\left(\left\|w_{d}\right\|_{\left[t_{2 i}\right]}\right), t_{2 i}-t_{2 j}\right) \\
& +\sum_{j=0}^{i-1} \beta\left(\gamma_{c}\left(\left\|w_{c}\right\|_{\left[t_{2 i+1}\right]}\right), t_{2 i}-t_{2 j+1}\right)+\gamma_{c}\left(\left\|w_{c}\right\|_{[t]}\right) .
\end{aligned}
$$

For any $(t, i) \in \mathscr{I}_{i}$, if $t \in I_{d i}=\mathscr{N}\left[t_{2 i+1}+1, t_{2(i+1)}\right]$, by (13) and condition (i), we get

$$
\begin{gathered}
\|x(t, i)\| \leq \beta\left(\left\|x_{0}\right\|, t-t_{0}\right)+\sum_{j=1}^{i} \beta\left(\gamma_{d}\left(\left\|w_{d}\right\|_{\left[t_{2 i}\right]}\right), t-t_{2 j}\right) \\
+\gamma_{d}\left(\left\|w_{d}\right\|_{[t]}\right)+\sum_{j=0}^{i} \beta\left(\gamma_{c}\left(\left\|w_{c}\right\|_{\left[t_{2 i+1}\right]}\right), t-t_{2 j+1}\right) \\
\leq \beta\left(\left\|x_{0}\right\|, t-t_{0}\right)+\sum_{j=1}^{i} \beta\left(\gamma_{d}\left(\left\|w_{d}\right\|_{\left[t_{2 i}\right]}\right), t_{2 i+1}-t_{2 j}\right) \\
+\gamma_{d}\left(\left\|w_{d}\right\|_{[t]}\right)+\sum_{j=0}^{i} \beta\left(\gamma_{c}\left(\left\|w_{c}\right\|_{\left[t_{2 i+1}\right]}\right), t_{2 i+1}-t_{2 j+1}\right) .
\end{gathered}
$$

Since $\beta \in \mathscr{K}^{+} \mathscr{L}^{+}$, thus, for any $a \in R_{+}$and $i, j \in N$ with $j \leq i$, we have $\beta\left(a, t_{2 i+1}-t_{2 j}\right) \leq \beta\left(a, t_{2 i}-t_{2 j}\right), \beta\left(a, t_{2 i}-\right.$ $\left.t_{2 j-1}\right) \leq \beta\left(a, t_{2 i}-t_{2 j}\right)$, and $\beta\left(a, t_{2 i+1}-t_{2 j+1}\right) \leq \beta\left(a, t_{2 i}-t_{2 j}\right)$.

Hence, it follows from (14)-(15) and (9) that

$$
\begin{aligned}
& \|x(t, i)\| \leq \beta\left(\left\|x_{0}\right\|, t-t_{0}\right)+\hat{\gamma}\left(\gamma_{c}\left(\left\|w_{c}\right\|_{[t]}\right)\right) \\
& \quad+\gamma_{c}\left(\left\|w_{c}\right\|_{[t]}\right)+\hat{\gamma}\left(\gamma_{d}\left(\left\|w_{d}\right\|_{[t]}\right)\right), t \in I_{c i}, i \in N \\
& \|x(t, i)\| \leq \beta\left(\left\|x_{0}\right\|, t-t_{0}\right)+\hat{\gamma}\left(\gamma_{c}\left(\left\|w_{c}\right\|_{[t]}\right)\right) \\
& \quad+\hat{\gamma}\left(\gamma_{d}\left(\left\|w_{d}\right\|_{[t]}\right)\right)+\gamma_{d}\left(\left\|w_{d}\right\|_{[t]}\right), t \in I_{d i}, i \in N .
\end{aligned}
$$

Let $\tilde{\gamma}_{c}(s)=\gamma_{c}(s)+\hat{\gamma}\left(\gamma_{c}(s)\right), \tilde{\gamma}_{d}(s)=\gamma_{d}(s)+\hat{\gamma}\left(\gamma_{d}(s)\right)$, for any $s \in R_{+}$, then, $\tilde{\gamma}_{c}, \tilde{\gamma}_{d} \in \mathscr{K}$. It follows from (16)-(17) that for any $(t, i) \in \mathscr{I}_{i}, i \in N$,

$$
\|x(t, i)\| \leq \beta\left(\left\|x_{0}\right\|, t-t_{0}\right)+\tilde{\gamma}_{c}\left(\left\|w_{c}\right\|_{[t]}\right)+\tilde{\gamma}_{d}\left(\left\|w_{d}\right\|_{[t]}\right),
$$

which means that HDS (1) has the first ISS property.

Case II: $S_{c i}$ has the ISS property while $S_{d i}$ may have no ISS property.

Theorem 3.2. Suppose $\Delta_{d}^{\text {sup }}<\infty$ and assume that there exist functions $V^{c}, V_{i}^{d} \in C\left[R_{+} \times R^{n}, R_{+}\right], i \in N$, such that the following conditions are satisfied:

(i) there exist $\mathscr{K}$-class functions $\varphi_{1}, \varphi_{2}$, such that

$$
\varphi_{1}(\|x\|) \leq V^{*}(t, x) \leq \varphi_{2}(\|x\|), V^{*}=V^{c} \text { or } V^{*}=V_{i}^{d} ;
$$

(ii) there exist $\mathscr{K} \mathscr{L}$-class function $\beta_{c}$, and $\mathscr{K}$-class function $\gamma_{c}$ such that for any $t \in I_{c i}, i \in N$,

$V^{c}(t, x(t, i)) \leq \beta_{c}\left(V^{c}\left(t_{2 i}, x\left(t_{2 i}, i\right)\right), t-t_{2 i}\right)+\gamma_{c}\left(\left\|w_{c}\right\|_{[t]}\right) ;$

(iii) there exist $\mathscr{K}$-class functions $\psi, \gamma_{d}$ with $\psi(s) \geq s$ and $\psi \in \mathscr{K}^{+}$, such that for any $t \in I_{d i}, i \in N$,

$$
V_{i}^{d}(t, x(t, i)) \leq \psi\left(V_{i}^{d}(t-1, x(t-1, i))\right)+\gamma_{d}\left(\left\|w_{d}\right\|_{[t-1]}\right) ;
$$

(iv) for any $i \geq 1, i \in N$, the solution $x(t)$ satisfies

$$
\begin{aligned}
& V^{c}\left(t_{2 i}, x\left(t_{2 i}, i\right)\right) \leq V_{i-1}^{d}\left(t_{2 i}, x\left(t_{2 i}, i-1\right)\right), \\
& V_{i-1}^{d}\left(t_{2 i-1}, x\left(t_{2 i-1}, i-1\right)\right) \leq V^{c}\left(t_{2 i-1}, x\left(t_{2 i-1}, i-1\right)\right) ;
\end{aligned}
$$

(v) there exists a function $\beta \in \mathscr{K}^{+} \mathscr{L}^{+}$satisfying, for any $a, s \in R_{+}$and any $m \in \mathscr{N}\left[0, \Delta_{d}^{\text {sup }}\right]$,

$$
\psi^{m}\left(\beta_{c}(a, s)\right) \leq \beta(a, m+s),
$$

and for any $a \in R_{+}$, there exists a $\mathscr{K}^{+}$-class function $\hat{\gamma}$ satisfying (9).

Then, HDS (1) has the first ISS property. 
Proof. Let $a_{i}=V_{i-1}^{d}\left(t_{2 i}, x\left(t_{2 i}, i-1\right)\right)$ for any $i \geq 1$, and for $i \in N$, let $v_{i}=\psi^{\Delta_{d i}}\left(\gamma_{c}\left(\left\|w_{c}\right\|_{\left[t_{2 i+1}\right]}\right)\right)+\gamma_{d}\left(\left\|w_{d}\right\|_{\left[t_{2(i+1)}\right]}\right)+$ $\psi\left(\gamma_{d}\left(\left\|w_{d}\right\|_{\left[t_{2(i+1)}\right]}\right)\right)+\cdots+\psi^{\Delta_{d i}-1}\left(\gamma_{d}\left(\left\|w_{d}\right\|_{\left[t_{2(i+1)}\right]}\right)\right)$. By conditions (ii)-(iii), it yields that

$$
\begin{aligned}
& a_{i+1} \leq \psi^{\Delta_{d i}}\left(V_{i}^{d}\left(t_{2 i+1}, x\left(t_{2 i+1}, i\right)\right)\right)+\tilde{\gamma}_{d i}\left(\left\|w_{d}\right\|_{\left[t_{2(i+1)}\right]}\right) \\
& \leq \psi^{\Delta_{d i}}\left(\beta_{c}\left(a_{i}, \Delta_{c i}\right)+\gamma_{c}\left(\left\|w_{c}\right\|_{\left[t_{2 i+1}\right]}\right)\right)+\tilde{\gamma}_{d i}\left(\left\|w_{d}\right\|_{\left[t_{2(i+1)}\right]}\right) \\
& \leq \beta\left(a_{i}, \Delta_{i}^{+}\right)+v_{i}, \quad i \geq 1, i \in N,
\end{aligned}
$$

where $\Delta_{i}^{+}=\Delta_{c i}+\Delta_{d i}, \quad \tilde{\gamma}_{d i}(s)=\gamma_{d}(s)+\psi\left(\gamma_{d}(s)\right)+\cdots+$ $\psi^{\Delta_{d i}-1}\left(\gamma_{d}(s)\right)$, for any $s \in R_{+}, i \in N$.

Since $\beta \in \mathscr{K}^{+} \mathscr{L}^{+}$, it derives from (23) and conditions (ii)-(iii) that

$a_{i+1} \leq \beta\left(V^{c}\left(t_{0}, x_{0}\right), \sum_{j=0}^{i} \Delta_{j}^{+}\right)+\sum_{j=0}^{i-1} \beta\left(v_{j}, \sum_{k=j+1}^{i} \Delta_{k}^{+}\right)+v_{i}, i \in N$.

Thus, it yields that

$$
\begin{aligned}
a_{i+1} & \leq \beta\left(V^{c}\left(t_{0}, x_{0}\right), t_{2(i+1)}-t_{0}\right) \\
& +\sum_{j=0}^{i-1} \beta\left(v_{j}, t_{2(i+1)}-t_{2(j+1)}\right)+v_{i}, i \in N .
\end{aligned}
$$

By (22), we have $\beta_{c}(a, s) \leq \beta(a, s)$ for any $a, s \in R_{+}$. Thus, for any $(t, i) \in \mathscr{I}_{i}$, if $t \in I_{c i}$, then, it follows from (20) and (25) that

$$
\begin{aligned}
& V^{c}(t, x(t, i)) \leq \beta\left(a_{i}, t-t_{2 i}\right)+\gamma_{c}\left(\left\|w_{c}\right\|_{[t]}\right) \\
& \leq \beta\left(V^{c}\left(t_{0}, x_{0}\right), t-t_{0}\right)+\sum_{j=0}^{i-1} \beta\left(v_{j}, t_{2 i}-t_{2(j+1)}\right) \\
& \quad+\gamma_{c}\left(\left\|w_{c}\right\|_{[t]}\right) .
\end{aligned}
$$

If $t \in I_{d i}$, then, by (25) and (20)-(21), we get

$$
\begin{aligned}
& V_{i}^{d}(t, x(t, i)) \leq \psi^{t-t_{2 i+1}}\left(V_{i}^{d}\left(t_{2 i+1}, x\left(t_{2 i+1}, i\right)\right)\right) \\
& \quad+\tilde{\gamma}_{d i}\left(\left\|w_{d}\right\|_{[t]}\right) \\
& \leq \psi^{t-t_{2 i+1}}\left(\beta_{c}\left(a_{i}, t_{2 i+1}-t_{2 i}\right)+\gamma_{c}\left(\left\|w_{c}\right\|_{\left[t_{2 i+1}\right]}\right)\right) \\
& \quad+\tilde{\gamma}_{d i}\left(\left\|w_{d}\right\|_{[t]}\right) \\
& \leq \beta\left(a_{i}, t-t_{2 i}\right)+\psi^{\Delta_{d}^{s u p}}\left(\gamma_{c}\left(\left\|w_{c}\right\|_{\left[t_{2 i+1}\right]}\right)\right)+\tilde{\gamma}_{d i}\left(\left\|w_{d}\right\|_{[t]}\right) \\
& \leq \\
& \quad \beta\left(V^{c}\left(t_{0}, x_{0}\right), t-t_{0}\right)+\sum_{j=0}^{i-1} \beta\left(v_{j}, t_{2 i}-t_{2(j+1)}\right) \\
& \quad+\psi^{\Delta_{d}^{s u p}}\left(\gamma_{c}\left(\left\|w_{c}\right\|_{\left[t_{2 i+1}\right]}\right)\right)+\tilde{\gamma}_{d i}\left(\left\|w_{d}\right\|_{[t]}\right) .
\end{aligned}
$$

Thus, by using (26)-(27) and condition (i), we obtain that for any $(t, i) \in \mathscr{I}_{i}, i \in N$,

$$
\varphi_{1}(\|x(t, i)\|) \leq \tilde{\beta}\left(\left\|x_{0}\right\|, t-t_{0}\right)+\tilde{\gamma}_{c}\left(\left\|w_{c}\right\|_{[t]}\right)+\tilde{\gamma}_{d}\left(\left\|w_{d}\right\|_{[t]}\right),
$$

where $\tilde{\beta}(a, s)=\beta\left(\varphi_{2}(a), s\right), \quad \tilde{\gamma}_{c}(s)=\hat{\gamma}\left(\psi^{\Delta_{d}^{s u p}}\left(\gamma_{c}(s)\right)\right)+$ $\psi^{\Delta_{d}^{s u p}}\left(\gamma_{c}(s)\right), \tilde{\gamma}_{d}(s)=\hat{\gamma}_{d}(s)+\hat{\gamma}\left(\hat{\gamma}_{c}(s)\right), \quad \hat{\gamma}_{d}(s)=\gamma_{d}(s)+$ $\psi\left(\gamma_{d}(s)\right)+\cdots+\psi^{\Delta_{d}^{s u p}-1}\left(\gamma_{d}(s)\right)$.

Hence, HDS (1) has the first ISS property.

Case III: $S_{d i}$ has the ISS property while $S_{c i}$ may have no ISS property.

Theorem 3.3. Suppose $\Delta_{c}^{\text {sup }}<\infty, \Delta_{d}^{\text {inf }}>0$ and assume that there exist functions $V^{c}, V_{i}^{d} \in C\left[R_{+} \times R^{n}, R_{+}\right], i \in N$, such that the conditions (i) and (iv) of Theorem 3.2 hold and the following conditions are satisfied:

(i) there exist $\mathscr{K}^{+} \mathscr{K}$-class function $\alpha_{c}$, and $\mathscr{K}$-class function $\gamma_{c}$ such that for any $t \in I_{c i}$,

$V^{c}(t, x(t, i)) \leq \alpha_{c}\left(V^{c}\left(t_{2 i}, x\left(t_{2 i}, i\right)\right), t-t_{2 i}\right)+\gamma_{c}\left(\left\|w_{c}\right\|_{[t]}\right) ;$

(ii) there exist $\mathscr{K}$-class functions $\psi, \gamma_{d}$ with $\psi \in \mathscr{K}^{+}$and $\psi(s)<s$ for any $s>0$, such that for any $t \in I_{d i}, i \in N$,

$$
V_{i}^{d}(t, x(t, i)) \leq \psi\left(V_{i}^{d}(t-1, x(t-1, i))\right)+\gamma_{d}\left(\left\|w_{d}\right\|_{[t-1]}\right) ;
$$

(iii) there exists a function $\beta \in \mathscr{K}^{+} \mathscr{L}^{+}$satisfying (9) and, for any $a, s \in R_{+}$and any $m \in \mathscr{N}\left[\Delta_{d}^{\text {inf }},+\infty\right)$,

$$
\left.\alpha_{c}\left(\psi^{m}(a), s\right)\right) \leq \beta(a, m+s) .
$$

Then, HDS (1) has the first ISS property.

Proof. By using the similar proof of Theorem 3.2, we can derive the result. The details are omitted here.

Remark 3.1. In Theorem 3.2, if $\beta_{c}(a, s)=a e^{-\alpha s}, \psi(s)=q s$ for some positive constants $\alpha>0, q>1$, and any $a, s \in R_{+}$, then, we define $\beta(a, s)=a e^{-\alpha^{*} s}$, where $\alpha^{*}$ satisfies $0<\alpha^{*}<$ $\alpha$ and

$$
\frac{\Delta_{c i}}{\Delta_{d i}} \geq \frac{\ln q+\alpha^{*}}{\alpha-\alpha^{*}}, i \in N
$$

Similarly, in Theorem 3.3, if $\alpha_{c}(a, s)=a e^{\alpha s}, \psi(s)=\tilde{q} s$ for some positive constants $\alpha>0, \tilde{q}<1$, and any $a, s \in R_{+}$, then, we define $\beta(a, s)=a e^{-\alpha^{*} s}$, where $\alpha^{*}$ satisfies $0<\alpha^{*}<$ $-\ln \tilde{q}$ and

$$
\frac{\Delta_{d i}}{\Delta_{c i}} \geq \frac{\alpha+\alpha^{*}}{-\left(\ln \tilde{q}+\alpha^{*}\right)}, i \in N .
$$

It follows from (32)-(33) and Theorem 3.2-3.3 that there exists the first ISS property for the whole HDS if any one of these two kinds of dynamics has the first ISS property and the dwell time on the dynamics with the ISS property is enough long.

Theorem 3.4. Suppose $0<\Delta_{\text {inf }} \leq \Delta_{\text {sup }}<+\infty$ and assume that there exist functions $V^{c}, V_{i}^{d} \in C\left[R_{+} \times R^{n}, R_{+}\right], i \in N$, such that the condition (iv) of Theorem 3.2 holds and the following conditions are satisfied:

(i) there exist positive constants $c_{1}, c_{2}, r>0$, such that

$$
c_{1}\|x\|^{r} \leq V^{*}(t, x) \leq c_{2}\|x\|^{r}, V^{*}=V^{c} \text { or } V^{*}=V_{i}^{d}, t \in R_{+} ;
$$

(ii) there exist $\mathscr{K}$-class function $\gamma_{c}$, and constants $p_{i}$ with $0<\underline{p} \leq\left|p_{i}\right| \leq \bar{p}, i \in N$, for some positive constants $\underline{p}, \bar{p}$, such that for any $t \in I_{c i}, i \in N$,

$$
D^{+} V^{c}(t, x(t, i)) \leq p_{i} V^{c}(t, x(t, i))+\gamma_{c}\left(\left\|w_{c i}(t)\right\|\right) ;
$$

(iii) there exist a $\mathscr{K}$-class function $\gamma_{d}$ and constants $q_{i}$ with $q_{i}>-1$ and $0<\underline{q} \leq\left|q_{i}\right| \leq \bar{q}, i \in N$, for some positive constants $\underline{q}, \bar{q}$, such that for any $t \in I_{d i}, i \in N$,

$$
\begin{aligned}
& V_{i}^{d}\left(t, f_{d i}\left(t-1, x(t-1, i), w_{d i}(t-1)\right)\right) \leq \\
& \left(1+q_{i}\right) V_{i}^{d}(t-1, x(t-1, i))+\gamma_{d}\left(\left\|w_{d i}(t-1)\right\|\right) ;(36)
\end{aligned}
$$

(iv) there exists a constant $\alpha>0$ such that

$$
\sigma_{i} \triangleq \Delta_{c i} p_{i}+\Delta_{d i} \ln \left(1+q_{i}\right) \leq-\alpha \Delta_{i}^{+}, i \in N .
$$


Then, HDS (1) has the first exponential ISS property.

Proof. Due to space limitations, the details are omitted here.

\section{B. The Second ISS Property of HDS}

In this part, we investigate the second ISS property of HDS (1) under two special cases: $w_{c} \equiv 0$, and $w_{d} \equiv 0$. Due to space limitations, we include only an outline for the proof. Theorem 3.5. Let $w_{d} \equiv 0$. Suppose $\Delta_{c}^{\text {sup }}<\infty, \Delta_{d}^{\text {inf }}>1$, and assume that there exist functions $V^{c}, V_{i}^{d} \in C\left[R_{+} \times R^{n}, R_{+}\right], i \in$ $N$, such that the conditions (i) and (iv) of Theorem 3.2 holds and the following conditions are satisfied:

(i) there exist $\mathscr{K}$-class functions $c_{1}, c_{2}, \gamma_{c} \in \mathscr{K}$ and function $p \in C\left[R_{+}, R_{+}\right]$, such that for any $t \in I_{c i}, i \in N$,

$$
\begin{aligned}
& D^{+} V^{c}(t, x(t, i)) \leq p(t) c_{1}\left(V^{c}(t, x(t, i))\right) \\
& +\gamma_{c}\left(\left\|w_{c}(t)\right\|\right) c_{2}(V(t, x(t, i))) ;
\end{aligned}
$$

(ii) there exists a $\mathscr{K}$-class function $\psi$ satisfying $\psi \in \mathscr{K}^{+}$ and $\psi(s)<s$ for all $s>0$, such that for any $t \in I_{d i}, i \in N$,

$$
\begin{aligned}
& V_{i}^{d}\left(t, f_{d i}\left(t-1, x(t-1, i), w_{d i}(t-1, i)\right)\right) \\
& \quad \leq \psi\left(V_{i}^{d}(t-1, x(t-1, i))\right),
\end{aligned}
$$

where for any $a \in R_{+}$, there exists a $\hat{\gamma} \in \mathscr{K}$ such that

$$
\sum_{i=0}^{\infty} \psi^{i}(a) \leq \hat{\gamma}(a)
$$

(iii) for any $i \in N$, the following inequality holds for any positive constants $z, \sigma>0$ with $0 \leq \sigma \leq \gamma_{c}\left(\left\|w_{c}\right\|_{\infty}\right)$,

$$
\int_{\psi(z)+\sigma}^{\psi^{\Lambda_{d i-1}(z)}} \frac{d s}{c(s)}+\int_{s \in I_{c i}}(p(s)+\sigma) d s \leq 0, \quad i \in N,
$$

where $c(s)=\max \left\{c_{1}(s), c_{2}(s)\right\}$ for any $s \in R_{+}$.

Then, HDS (1) has the second ISS property.

Proof. Let $m_{i}^{c}(t)=V^{c}(t, x(t, i)), m_{i}^{d}(t)=V_{i}^{d}(t, x(t, i))$, for any $(t, i) \in \mathscr{I}_{i}, i \in N$, and $a_{i}=V^{c}\left(t_{2 i+1}, x\left(t_{2 i+1}, i\right)\right), v_{i}(t)=$ $\gamma_{c}\left(\left\|w_{c}\right\|_{[t]}\right)$, for $(t, i) \in \mathscr{I}_{i}$, and $v_{i}=v_{i}\left(t_{2 i+1}\right), i \in N$.

Claim 1 $1^{\circ}$ : for any $(t, i) \in \mathscr{I}_{i}$, if $t \in I_{c i}$, we claim that

$$
m_{i}^{c}(t) \leq \psi^{i}\left(a_{0}\right)+\sum_{j=0}^{i-1} \psi^{j}\left(\tilde{v}_{i-j}\right), t \in I_{c i}, i \in N,
$$

where for any $0 \leq k \leq i, \tilde{v}_{k}=\left\{\begin{array}{l}v_{i}(t), k=i ; \\ v_{k}, k<i .\end{array}\right.$

Claim $2^{\circ}$ : if $t \in I_{d i}=\mathscr{N}\left[t_{2 i+1}+1, t_{2(i+1)}\right]$, we claim that

$$
m_{i}^{d}(t) \leq \psi^{i}\left(a_{0}\right)+\sum_{j=0}^{i-1} \psi^{j}\left(\tilde{v}_{i-j}\right), t \in I_{d i}, i \in N .
$$

Claim $3^{\circ}$ : by Lemma 2.1 , there exists a function $\hat{\beta} \in$ $\mathscr{K} \mathscr{K}$ such that

$$
a_{0}=m_{0}^{c}\left(t_{1}\right) \leq \hat{\beta}\left(m_{0}^{c}\left(t_{0}\right), t_{1}-t_{0}\right) .
$$

Hence, by Claims $1^{\circ}-3^{\circ}$, let $\beta(a, i)=\psi^{i}\left(\hat{\beta}\left(\varphi_{2}(a), \Delta_{c}^{\text {sup }}\right)\right)$, for any $a \in R_{+}, i \in N$, and $\tilde{\gamma}(s)=\hat{\gamma}\left(\gamma_{c}(s)\right)$ for any $s \in R_{+}$, then, $\beta \in \mathscr{K} \mathscr{D}, \tilde{\gamma} \in \mathscr{K}$, and for any $(t, i) \in \mathscr{I}_{i}, i \in N$,

$$
\varphi_{1}(\|x(t, i)\|) \leq \beta\left(\left\|x_{0}\right\|, i\right)+\tilde{\gamma}_{c}\left(\left\|w_{c}\right\|_{[t]}\right) .
$$

Hence, HDS (1) has the second ISS property.

Theorem 3.6. Let $w_{c} \equiv 0$. Suppose $\Delta_{d}^{s u p}<\infty$ and assume that there exist functions $V^{c}, V_{i}^{d} \in C\left[R_{+} \times R^{n}, R_{+}\right], i \in N$, such that the conditions (i) and (iv) of Theorem 3.2 holds and the following conditions are satisfied:

(i) there exist functions $c \in \mathscr{K}, p \in C\left[R_{+}, R_{+}\right]$, such that for any $t \in I_{c i}, i \in N$,

$$
D^{+} V^{c}(t, x(t, i)) \leq-p(t) c\left(V^{c}(t, x(t, i))\right) ;
$$

(ii) there exist $\mathscr{K}$-class functions $\psi, \gamma_{d}$ with $\psi \in \mathscr{K}^{+}$and $\psi(s) \geq s$ for any $s \in R_{+}$, such that for any $t \in I_{d i}, i \in N$,

$$
\begin{aligned}
& V_{i}^{d}\left(t, f_{d i}\left(t-1, x(t-1, i), w_{d i}(t-1)\right)\right) \\
& \leq \psi_{i}\left(V_{i}^{d}(t-1, x(t-1, i))\right)+\gamma_{d}\left(\left\|w_{d i}(t-1)\right\|\right)
\end{aligned}
$$

(iii) there exists a increasing function $\tilde{\psi}$ with $\tilde{\psi}^{-1} \in \mathscr{K}^{+}$and $\tilde{\psi}(s)>s$ for any $s>0$, such that for any positive constant $z>0$,

$$
-\int_{s \in I_{c i}} p_{i}(s) d s+\int_{z}^{\tilde{\psi}\left(\psi_{i}^{\Delta}{ }^{\Delta i}(z)\right)} \frac{d s}{c(s)} \leq 0, i \in N,
$$

where for any $a \in R_{+}$, there exists a $\mathscr{K}$-class function $\hat{\gamma}$ such that $\sum_{i=0}^{+\infty} \tilde{\psi}^{-i}(a) \leq \hat{\gamma}(a)$.

Then, HDS (1) has the second ISS property.

Proof. It can be derived by using the similar proof as in Theorem 3.5. The details are thus omitted.

Remark 3.2. Similar results on dwell time as in Remark 3.1 can be derived for the second ISS property of HDS. Moreover, one can see from the results and conditions of Theorems 3.5-3.6 that the second ISS property for the whole HDS exists when one kind of dynamics without external inputs in the HDS has a stability property while the other one with external inputs has no stability and ISS properties.

\section{EXAMPLE}

In this section, we give one example for illustration.

Example 4.1. Consider the HDS:

$$
\begin{gathered}
\dot{x}=f_{c}(t, x)+w_{c}(t)=A_{c}(t) x+w_{c i}(t), \quad t \in I_{c i} ; \\
x(t)=A_{d i}(t-1) x(t-1)+\varphi_{d i}(t-1, x(t-1)) \\
+w_{d i}(t-1), \quad t \in I_{d i}, i \in N,
\end{gathered}
$$

where $A_{c}(t)=\left(\begin{array}{ccc}1 & 0 & 0 \\ 0 & -0.1 & 0.1\left(1-e^{-t}\right) \\ 0 & 0 & 0.5\end{array}\right)$, and $A_{d i}(t)=$ $\left(\begin{array}{ccc}0.4+0.1 \sin (i) & 0 & 0 \\ 0 & 0.1 & 0.1 \sin (i) \\ 0.1\left(1-e^{-t}\right) & 0 & 0.5 \cos (i)\end{array}\right), \quad$ and $\quad \varphi_{d i}(t, x(t))=$ $\frac{1}{4}\left(\frac{x_{1}(t)}{1+\sin ^{2} i+\|x(t)\|^{2}} \quad x_{2}(t) \sin \left(x_{3}(t)\right) \quad x_{3}(t) \cos \left(x_{3}(t)\right)\right)^{T}$.

Assume that $\left\|w_{c}\right\|_{\infty}<\infty,\left\|w_{d}\right\|_{\infty}<\infty$ and the time intervals: $I_{c i}=\left[t_{2 i}, t_{2 i+1}\right]$, and $I_{d i}=\mathscr{N}\left[t_{2 i+1}+1, t_{2(i+1)}\right]$ are chosen as: $t_{0}=0 ; t_{2 i+1}=t_{2 i}+1.5 ; t_{2(i+1)}=t_{2 i+1}+6, i \in N$.

Let $V(t, x)=\|x\|$, we get

$$
\begin{aligned}
& D^{+} V \leq p V+d_{1}\left\|w_{c}\right\|_{[t]}, \quad t \in I_{c i} ; \\
& V(t+1, x(t+1)) \leq\left(1+q_{i}\right) V(t, x(t))+d_{2}\left\|w_{d}\right\|_{[t]}, t \in I_{d i},
\end{aligned}
$$


where $p=0.5, q_{i}=-0.25, d_{1}=d_{2}=1$. Thus, we get

$$
p \Delta_{c i}+\Delta_{d i} \ln \left(1+q_{i}\right) \leq-0.0301\left(\Delta_{c i}+\Delta_{d i}\right) .
$$

By Theorem 3.4, we get that the HDS has the first exponential ISS property. Moreover, we can get that

$$
\begin{aligned}
& \|x(t, i)\| \leq 2.5487\left\|x_{0}\right\| e^{-0.0301 t}+6.9634\left\|w_{c}\right\|_{[t]} \\
& \quad+8.4680\left\|w_{d}\right\|_{[t]}, \quad(t, i) \in \mathscr{I}_{i}, i \in N .
\end{aligned}
$$

In the simulation, we take the initial condition $t_{0}=$ $0, x_{0}=(1,3-5)^{T}$. The external disturbance inputs are in the form of: $w_{c}(t)=\operatorname{rand}(1)(-0.1,-0.1,0.1)^{T}, w_{d}(t)=$ $0.5(\sin (t), \sin (t), \cos (t))^{T}, t \in R_{+}$, where $\operatorname{rand}(1)$ is a random number satisfying $0 \leq \operatorname{rand}(1) \leq 1$. The result of numerical simulations is given in Fig.1.

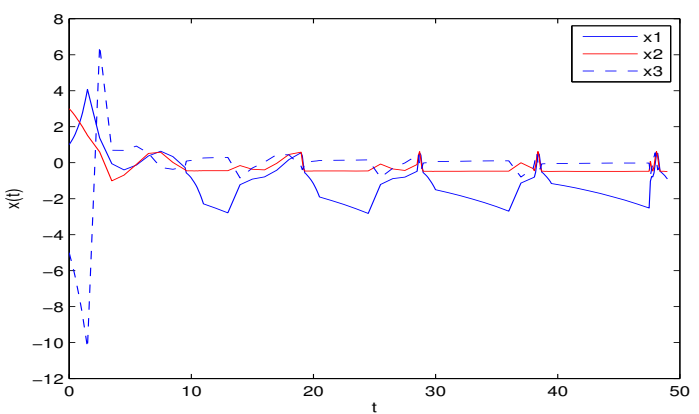

Fig.1. The first ISS property of HDS (49).

\section{REFERENCES}

[1] E.D. Sontag, "Smooth stabilization implies coprime factorization," IEEE Trans. on Automatic Control, 34, 1989, pp.435-443.

[2] E. D. Sontag, Y. Wang, "On characterization of the input-to-state stability property," Systems \& Control Letters, 24, 1995, pp.351-359.

[3] Z-P. Jiang, Y. Wang, "Input-to-state stability for discrete-time nonlinear systems," Automatica, 37, 2001, pp. 857-869.

[4] Z-P. Jiang, I. Mareels, D. J. Hill and J. Huang, "A unifying framework for global regulation via nonlinear output feedback: from ISS to integral ISS," IEEE Trans Auto Control, 49(4), 2004, pp. 549-562.

[5] D. Nešić, and A. Teel, "Input-output stability properties of networked control systems," IEEE Trans. on Automatic Control, 49, 2004, pp.1650-1667.

[6] A.R. Teel, "Connections between Razumikhin-type theorems and the ISS nonlinear small gain theorem," IEEE Trans. on Automatic Control, 43, 1998, pp.960-964.

[7] D. Nešić, and A. Teel, "Input-to-state stability for nonlinear timevarying systems via averaging," Mathematical of Control, Signals and Systems, 14, 2001, pp.257-280.

[8] D. Nešić, D. S. Laila, "A note on input-to-state stabilization for nonlinear sampled-data systems," IEEE Trans. on Automatic Control, 47(7), 2002, pp.1153-1158.

[9] D.S. Laila, and A. Astolfi, "Input-to-state stability for discrete-time time-varying systems with applications to robust stabilization of systems in power form," Automatica, 41, 2005, pp.1891-1903.

[10] S.D. Huang, R. James, D. Nešić, and P.M. Dower, "A unified approach to controlled design for achieving ISS and related properties," IEEE Trans. on Automatic Control, 50, 2005, pp.1681-1697.

[11] J.P. Hespanha, D. Liberzon, and A.R. Teel, "On input-to-state stability of impulsive systems," Proceeding of the 44th IEEE Conferences on Decision and Control, 2005, pp.3992-3997.

[12] J.P. Hespanha, D. Liberzon, and A.R. Teel, "Lyapunov conditions for input-to-state stability of impulsive systems," Automatica, 44, 2008, pp.2735-2744.

[13] D. Liberzon, and Nešić, "Input-to-state stabilization of linear systems with quantized state measurememts," IEEE Trans. on Automatic Control, 52, 2007, pp.767-781.
[14] P. Pepe, and Z.-P. Jiang, "A Lyapunov-Krasovskii methodology for ISS and iISS of time-delay systems," Syst. Control Lett., 55(12), 2006, pp.1006-1014.

[15] E. Mazenc, M. Michel, and Z. Lin, "On input-to-state stability for nonlinear systems with delayed feedbacks," Proc. Autom. Control Conf., New York, NY, 2007, pp.4804-4809.

[16] N. Yeganefar, P. Pepe, and M. Dambrine, "Input-to-state stability of time-delay systems: a link with exponential stability," IEEE Trans on Automatic Control, 53(6), 2008, pp.1526-1531.

[17] Z-P. Jiang, A. R. Teel, and L. Praly, "Small-gain theorem for ISS systems and applications," Mathematics of Control Signals and Systems, 7(2), 1994, pp.95-120.

[18] Z-P. Jiang, I. M. Y. Mareels, and Y. Wang, "A Lyapunov formulation of the nonlinear small-gain theorem for interconnected ISS systems," Automatica, 30(8), 1996, pp.1211-1215.

[19] C.H. Cai, and A.R. Teel, "Characterizations of input-to-state stability for hybrid systems," Syst. Control Lett., 58, 2009, pp.47-53.

[20] A. R. Teel, L. Praly, "A smooth Lyapunov function from a class$\mathscr{K} \mathscr{L}$ estimate invovling two positive semidefinite functions," ESIAM Control, Optim. Calc. Var., 5, 2000, pp.313-367.

[21] C.H. Cai, A.R. Teel, and R. Goebel, "Smooth Lyapunov functions for hybrid systems Part I: Existence is equivalent to robustness," IEEE Trans. on Automatic Control, 52(7), 2007, pp.1264-1277.

[22] C.H. Cai, A.R. Teel, and R. Goebel, "Smooth Lyapunov functions for hybrid systems part II: (Pre)asymptotically stable compact sets," IEEE Trans. on Automatic Control, 53(3), 2008, pp.734-748.

[23] L. Moreau, "Stability of multiagent systems with time-dependent communication links," IEEE Trans. on Automatic Control, 50(2), 2005, pp.169-182.

[24] R. Goebel, A.R. Teel, "Solutions to hybrid inclusions via set and graphical convergence with stability theory applications," Automatica, 42(4), 2006, pp.573-587.

[25] R.G. Sanfelice, R. Goebel, and A.R. Teel, "Invariance principles for hybrid systems with connections to detectability and asymptotic stability," IEEE Trans. on Automatic Control, 52(12), 2007, pp.22822297.

[26] V. Lakshmikantham, D.D. Bainov and P.S. Simeonov, Theory of impulse differential equations, World Scientific, Singapore, 1989.

[27] A.N. Michel, "Recent trends in the stability analysis of hybrid dynamical systems," IEEE Transactions on Automatic Control, 45(1), 1999, pp.120-134.

[28] H. Ye, A.N. Michel, and L. Hou, "Stability theory for hybrid dynamical systems," IEEE Transactions on Automatic Control, 43(4), 1998, pp. $461-474$

[29] M. S. Branicky, "Multiple Lyapunov functions and other analysis tools for switched and hybrid systems", IEEE Trans. on Automatic Control, 43(4), 1998, pp:475-482.

[30] D. Liberzon, Switching in Systems and Control. Boston, MA Birkhäuser, 2003.

[31] J.P. Hespanha, A. S. Morse, "Stability of switched systems with average dwell-time," Proceeding of the 38th IEEE Conferences on Decision and Control, (New York: IEEE), pp.2655-2660, 1999.

[32] J. P. Aubin, J. Lygeros, M. Quinncampoix, S. Sastry, and N. Seube, "Impulse differential inclusions: A viability approach to hybrid systems", IEEE Trans. on Automatic Control, 47(1), 2002, pp.1-20.

[33] J. Lygeros, K. H. Johansson, S. N. Simić, J. Zhang, and S. Sastry, "Dynamical properties of hybrid automata", IEEE Trans. on Automatic Control, 48(2), 2003, pp.2-17.

[34] G. Xie and L. Wang, "Stability and stabilization of switched impulsive systems," Proc. the 2006 American Control Conference, Minneapolis, MN, 2006, pp.4406-4411.

[35] W.M. Haddad, V-S. Chellaboina, S.G. Nersesov, Impulsive and Hybrid Dynamical Systems, Stability, Dissipativity, and Control. Princeton, NJ: Princeton University Press, 2006.

[36] Z.G. Li, Y.C. Soh, and C.Y. Wen, "Robust stability of a class of hybrid nonlinear systems," IEEE Trans. on Automatic Control, 46, 2002, pp.897-903.

[37] B. Liu, H. J. Marquez, "Quasi-exponential ISS for discrete impulsive hybrid systems," Int. J. Control, 80(4), 2007, pp. 540-554.

[38] B. Liu, "Stability of solutions for stochastic impulsive systems," IEEE Trans on Automatic Control, 53(9), 2008, pp.2128-2133.

[39] B. Liu, D. J. Hill, "Input-to-state stability for discrete delay systems via Razumikhin technique," Syst. Control Lett., 58, 2009, pp.567-575. 